\title{
Solving an Ill-Posed Cauchy Problem for a Two-Dimensional Parabolic PDE with Variable Coefficients Using a Preconditioned GMRES Method
}

Zohreh Ranjbar and Lars Eldén

\author{
Linköping University Post Print
}

\section{Tweet}

N.B.: When citing this work, cite the original article.

Original Publication:

Zohreh Ranjbar and Lars Eldén, Solving an Ill-Posed Cauchy Problem for a Two-Dimensional Parabolic PDE with Variable Coefficients Using a Preconditioned GMRES Method, 2014, SIAM Journal on Scientific Computing, (36), 5, B868-B886.

http://dx.doi.org/10.1137/130951166

Copyright: Society for Industrial and Applied Mathematics http://www.siam.org/

Postprint available at: Linköping University Electronic Press http://urn.kb.se/resolve?urn=urn:nbn:se:liu:diva-111837 


\title{
SOLVING AN ILL-POSED CAUCHY PROBLEM FOR A TWO-DIMENSIONAL PARABOLIC PDE WITH VARIABLE COEFFICIENTS USING A PRECONDITIONED GMRES METHOD*
}

\author{
ZOHREH RANJBAR $^{\dagger}$ AND LARS ELDÉN ${ }^{\dagger}$
}

\begin{abstract}
The sideways parabolic equation (SPE) is a model of the problem of determining the temperature on the surface of a body from the interior measurements. Mathematically it can be formulated as a noncharacteristic Cauchy problem for a parabolic partial differential equation. This problem is severely ill-posed in an $L_{2}$ setting. We use a preconditioned generalized minimum residual method (GMRES) to solve a two-dimensional SPE with variable coefficients. The preconditioner is singular and chosen in a way that allows efficient implementation using the FFT. The preconditioner is a stabilized solver for a nearby problem with constant coefficients, and it reduces the number of iterations in the GMRES algorithm significantly. Numerical experiments are performed that demonstrate the performance of the proposed method.
\end{abstract}

Key words. Cauchy problem, inverse problem, ill-posed, iterative methods, GMRES preconditioning, singular preconditioner, parabolic PDE, FFT

AMS subject classifications. 35K05, 35K20, 35K67, 35R05, 35R30, 65T50, 65R20, 65R32, 65T40, 65F08, 65F10, 65N20, 65N06, 65F22, 65F10, 15A18, 65N21, 65N22

DOI. $10.1137 / 130951166$

1. Introduction. In several heat transfer situations $[1,9]$ it is required to determine the surface temperature of a body, where the surface itself is inaccessible to measurements. Industrial applications in two and three space dimensions include metal quench cooling [41], the monitoring of furnace linings [40, 39], and boiling heat transfer [10]. In the time-dependent case the problem can be formulated as a Cauchy problem for a parabolic partial differential equation, which we call a sideways parabolic equation (SPE). ${ }^{1}$ Often the SPE has variable coefficients $[40,10,3]$, for instance, when two materials are involved. Thus we consider the following twodimensional (2D) SPE, ${ }^{2}$

$$
\begin{array}{rlrl}
u_{t} & =\left(a(x, y) u_{x}\right)_{x}+\left(b(x, y) u_{y}\right)_{y}, & 0<x<1, & 0<y<1, \quad 0 \leq t \leq 1 \\
u(x, y, 0) & =0, & & 0 \leq x \leq 1, \quad 0 \leq y \leq 1 \\
u_{y}(x, 0, t) & =0, \quad u(x, 1, t)=0, & & 0 \leq x \leq 1, \quad 0 \leq t \leq 1 \\
u(1, y, t) & =g(y, t), & & 0 \leq y \leq 1, \quad 0 \leq t \leq 1 \\
u_{x}(1, y, t) & =0, & 0 \leq y \leq 1, \quad 0 \leq t \leq 1,
\end{array}
$$

where $u(0, y, t)=f(y, t)$ is sought from the data at the right boundary; see Figure 1 . We consider both Cauchy data $g(y, t)$ and solution $f(y, t)$ to be $L^{2}$ functions in $(0,1) \times$ $(0,1)$, and the coefficient functions are in $L^{\infty}$. It is assumed that for exact data $g$ the function $f$ exists and the function $u(x, y, t)$ is in $L^{2}((0,1) \times(0,1) \times(0,1))$.

This type of problem is severely ill-posed [25, 21]: A small perturbation of the data can induce a large error in the solution. Due to ill-posedness and unavoidable noise

*Submitted to the journal's Computational Methods in Science and Engineering section January 2, 2014; accepted for publication (in revised form) August 8, 2014; published electronically October 21, 2014.

http://www.siam.org/journals/sisc/36-5/95116.html

${ }^{\dagger}$ Department of Mathematics, Linköping University, SE-581 83 Linköping, Sweden (zohreh. ranjbar@sics.se, lars.elden@liu.se).

${ }^{1}$ In some literature [1] it is called the inverse heat conduction problem.

${ }^{2}$ In the numerical experiments in section 4 we demonstrate that also other boundary conditions can be used, e.g., homogeneous Dirichlet. Also we are considering solutions in the classic sense.

B868 

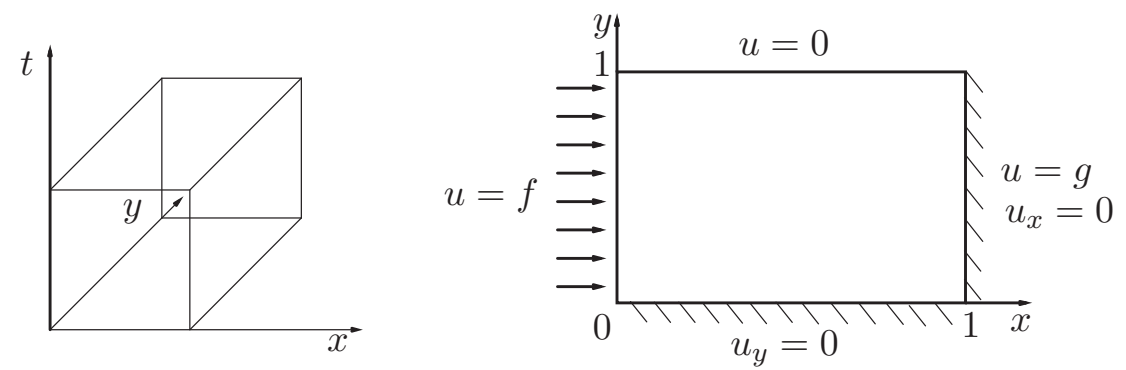

FIG. 1. 2D sideways parabolic problem. Right: cross section at $t=0$.

(measurement and rounding errors) in the data we must use a regularization method to compute a stabilized solution that is less sensitive to the noise. By regularization the noise is filtered out at the cost of restricting the set of admissible solutions.

Most numerical methods for the SPE problem have been restricted to one-dimensional (1D) models, and only a few results are available in the $2 \mathrm{D}$ case $[15,27,32,36$, $41,26,33]$. To our knowledge, there are no papers presenting numerical algorithms for sideways parabolic Cauchy problems in two dimensions with variable coefficients. Ill-posed problems can be solved by Tikhonov regularization if they can be reformulated as integral equations. This is not feasible here, because the kernel function is not known for variable coefficients and impractical to compute. Therefore we suggest an iterative method where the main computations are the solution of well-posed problems. Thus in this paper we propose a method for solving numerically a 2D SPE with variable coefficients, discrete in space, using a preconditioned Krylov subspace method, GMRES (generalized minimum residual) [38]. The properties of Krylov methods applied to ill-posed problems have recently been studied in several papers $[24,6,7,8,20,23,4]$; also preconditioned GMRES has been proposed [20]. It has been reported [23] that without preconditioner GMRES fails to solve even 1D sideways parabolic problems. We here show that it is possible to construct an efficient preconditioner for the 2D case using a nearby equation with constant coefficients. However, such an equation would also be ill-posed. In order to stabilize the preconditioner we use a low-rank approximation, thus making the preconditioned system singular. The use of GMRES for solving such systems was proposed in [34, 35] and recently analyzed in [13]. We demonstrate that with this preconditioner GMRES gives a good approximate solution to problems in two dimensions in a small number of iterations.

The outline of the paper is as follows. In section 2, we give some background on the preconditioned GMRES (PGMRES) method and discuss the effect of a singular preconditioner. In section 3 we develop a Fourier-based, stabilized preconditioner which is derived from the 2D SPE problem with constant coefficients. In section 4 we provide some numerical results to illustrate that our schemes work well. Concluding remarks are given in section 5 .

Throughout the paper $\|\cdot\|$ denotes the discrete $L^{2}$ norm.

2. Singularly preconditioned GMRES for discrete ill-posed problems. In order to motivate the use of singularly PGMRES for ill-posed problems in section 2.2, we first describe PGMRES for well-posed problems in section 2.1.

2.1. Preconditioned GMRES for well-posed problems. Krylov subspace methods are methods of choice for the iterative solution of large and sparse linear systems $A x=b, A \in \mathbb{R}^{n \times n}$, arising in the discretization of well-posed problems for 
PDEs. The GMRES method by Saad and Schultz [38] is a standard Krylov subspace method for problems with nonsymmetric matrix $A$. GMRES with right preconditioner $M$ is given below; for details, see [37, p. 270].

GMRES with Right Preconditioning

1. Compute $r_{0}=b, \beta=\left\|r_{0}\right\|_{2}$, and $v_{1}=r_{0} / \beta$

2. for $j=1, \ldots, m$,

Compute $w:=A M^{-1} v_{j}$

for $i=1, \ldots, j$ do

$h_{i, j}:=w^{T} v_{i}$

end

$w:=w-h_{i, j} v_{i}$

Compute $h_{j+1, j}=\|w\|_{2}$ and $v_{j+1}=w / h_{j+1, j}$

end

3. Define $V_{m}:=\left[v_{1}, \ldots, v_{m}\right], H_{m}=\left\{h_{i, j}\right\}_{1 \leq i \leq j+1,1 \leq j \leq m}$

4. Compute $y_{m}=\operatorname{argmin}_{y}\left\|\beta e_{1}-H_{m} y\right\|_{2}$ and $x_{m}=M^{-1} V_{m} y_{m}$

The algorithm generates an orthonormal basis $\left[v_{1}, v_{2}, \ldots, v_{m}\right]=V_{m}$ of the Krylov subspace

$$
\mathcal{K}_{m}(\widehat{A}, b)=\operatorname{span}\left\{b, \widehat{A} b, \widehat{A}^{2} b, \ldots, \widehat{A}^{m-1} b\right\},
$$

where $\widehat{A}=A M^{-1}$. Note that it is not necessary for the matrix $\widehat{A}$ to be explicitly available, only the action of the operator to a vector, $\psi=\widehat{A} \phi$, is needed.

Unpreconditioned GMRES ( $M$ equal to the identity matrix) usually does not converge fast enough to be useful for solving large systems. The purpose of preconditioning is to improve the rate of convergence by compressing the spectrum of $\widehat{A}$ (as compared to that of $A$ ). To that end, one tries to find $M$ that is a good approximation of $A$, so that $\widehat{A}$ becomes close to the identity. For the method to be efficient it is also required that the solution of a system $M z=w$ can be computed quickly.

2.2. Singularly preconditioned GMRES for ill-posed problems. It is well known that unpreconditioned Krylov subspace methods give good solutions of illposed problems after a rather small number of steps, and that after a certain number of steps the error in the computed solution starts to grow, the so-called "semiconvergence" behavior; cf. [30, p. 259]. This is due to the fact that in its first few steps the Krylov method approximates mainly solution components associated with the "wellposed part." Gradually the "ill-posed part" starts to influence the solution, and then the solution explodes [13]. However, unpreconditioned GMRES does not work for solving the SPE problem [23, 13].

The SPE problem (1.1) can be written formally as a linear operator equation,

$$
K f=g,
$$

where $K$ is a fine discretization of a certain Volterra integral operator of the first kind. For general coefficients $a(x, y)$ and $b(x, y)$ we cannot give an explicit expression for the kernel function in the Volterra integral reformulation. Therefore any standard method, like Tikhonov regularization and truncated singular value decomposition (SVD) (see, e.g., [18] for an overview), based on writing the problem explicitly as a matrix equation $K f=g$ is impractical to use numerically for Cauchy problems with variable coefficients. Instead we use GMRES, where in each iteration the action of the operator $K$ is computed by solving a well-posed parabolic problem; see section 2.3.

Copyright (C) by SIAM. Unauthorized reproduction of this article is prohibited. 
If we were to precondition (2.1) as in section 2.1 we would try to find an operator $M$ that approximates $K$ well. Since we are solving an ill-posed problem, where the inverse $K^{-1}$ is very large, a good approximation $M$ would also have $M^{-1}$ very large. However, in the context of ill-posed problems, there is no need to approximate the "high frequency" part of $K$, because it is that part that accounts for the ill-conditioning. Therefore we investigate in this paper a preconditioner, derived from the corresponding problem with constant coefficients, where the high frequency components are eliminated by regularizing and truncating a Fourier expansion; this leads to a singular preconditioner. Preconditioners using fast FFT-based solvers for the corresponding problem with constant coefficients are standard for well-posed elliptic and hyperbolic problems; see, e.g., [5, p. 7], [22], but to our knowledge they have not been used for ill-posed parabolic problems. In particular, singular preconditioners have not been used before. Thus we solve the problem

$$
K M_{r}^{\dagger} y=g, \quad f=M_{r}^{\dagger} y,
$$

where $M_{r}^{\dagger}$ is a rank- $r$ approximation of $K^{-1}$, constructed so that the high frequency components of the solution are omitted.

The applicability of this type of truncated and singular preconditioner to the solution of ill-posed parabolic Cauchy problems was investigated in $[34,35]$. There we used GMRES with singular preconditioners for a 1D SPE, and found experimentally that it also works well for 2D problems. In [13] we analyzed the solution of almost singular linear systems by GMRES, and the use of singular preconditioners. We first showed that if $g_{e}$ is an exact data vector, and $f_{e}=K^{-1} g_{e}$ the corresponding solution, then we have

$$
\left\|f_{e}-f_{m}\right\| \leq\left\|S f_{e}\right\|+\left\|M_{r}^{\dagger}\left(K M_{r}^{\dagger}\right)^{+}\left(g_{e}-g\right)\right\|,
$$

where $f_{m}=M_{r}^{\dagger} y$, and $y$ is the minimum norm solution of $\min _{y}\left\|\left(K M_{r}^{\dagger}\right) y-g\right\|$. If the preconditioner is good, then $S$ is a projection onto the high frequency part of the solution. This estimate is analogous to those in the proofs of Proposition 3.7 and Theorem 3.26 in [14], where with an assumption about the smoothness of the exact solution $f_{e}$ and with a suitable regularization parameter choice rule (e.g., the discrepancy principle), continuous dependence on the data is proved. From the estimate we see that the preconditioner itself induces regularization, and thus stabilizes the problem.

In [13] we further showed that if the preconditioner is a good approximation for the low frequency part of the solution, then after a small number of iterations a stopping criterion based on the discrepancy principle [28] will be satisfied, and we have obtained a good approximate regularized solution. In fact, the preconditioner can be thought of as an approximate regularized solution operator, whose action is corrected by a few iterations of GMRES.

2.3. The operator $K$ for the 2D SPE. As we have remarked earlier, the operator $K$ is not known in the case of nonconstant coefficients. In the iterative solution of (2.1) by GMRES we need to repeatedly apply a discretization of the operator $K$ to a vector, $\psi=K \phi$. This corresponds to solving the following wellposed initial boundary value problem,

$$
\begin{array}{rlrl}
u_{t} & =\left(a(x, y) u_{x}\right)_{x}+\left(b(x, y) u_{y}\right)_{y}, & & 0<x<1, \quad 0<y<1, \quad 0 \leq t \leq 1 \\
u(x, y, 0) & =0, & & 0 \leq x \leq 1, \quad 0 \leq y \leq 1 \\
u(0, y, t) & =\phi(y, t), & & 0 \leq y \leq 1, \quad 0 \leq t \leq 1 \\
u_{x}(1, y, t) & =0, & 0 \leq y \leq 1, \quad 0 \leq t \leq 1 \\
u_{y}(x, 0, t) & =0, \quad u(x, 1, t)=0, & & 0 \leq x \leq 1, \quad 0 \leq t \leq 1
\end{array}
$$


and evaluating the solution at $x=1$ to get $\psi(t)$. This can be done using any standard method for the solution of a $2 \mathrm{D}$ parabolic equation. For instance, one may use a method of lines: discretize the space derivatives, leading to a system of ODEs $U_{t}=$ $B U$, where the matrix $B$ is large and sparse. The ODE is then solved using an algorithm for stiff ODEs [16]. In this procedure one may take full advantage of the fact that the matrix $B$ is sparse.

The well-known discrepancy principle [28] is used to determine when to terminate the iteration process; see [8] for a discussion of this stopping criterion together with GMRES. We stop the iteration when

$$
\left\|K f^{(k)}-g_{\delta}\right\| \leq \delta .
$$

An important feature of right PGMRES is that it is possible to obtain the residual norm of the approximate solution $f^{(k)}$ without explicitly computing $f^{(k)}$; cf. [37, pp. 169, 270].

3. The 2D SPE with constant coefficients. In this section we consider the case when the coefficients in (1.1) are constant. This analysis gives insight into the nature of the ill-posedness for both constant and nonconstant coefficients. It is also crucial for the construction of the preconditioner. Lemma 3.1 (from [36]) gives important information on the properties of the Cauchy problem, and it allows us to derive an explicit solution formula (3.12), from which the preconditioner is constructed in section 3.2 .

3.1. Analysis and solution formula. The ill-posedness of problem (1.1) can be seen by solving the problem with $a=b=1$ in the Fourier domain. The analysis is based on Fourier transforms and is standard; for completeness, we give it in Appendix A.

When the coefficients are constant then one can derive a 2D integral equation corresponding to (1.1), and apply Tikhonov regularization in one spatial dimension.

LEMma 3.1 (see [36]). The explicit representation of the solution of (1.1), with constant coefficients $a$ and $b$, in terms of the unknown function $u(0, y, t)=f(y, t)$ is

$$
u(x, y, t)=\int_{0}^{t} a \sum_{n, j=0}^{\infty}(-1)^{n} \nu_{n} \exp \left(-\left(a \nu_{n}^{2}+b \mu_{j}^{2}\right)(t-s)\right) \Psi_{n j}(x, y) f_{j}(s) d s
$$

where

and

$$
\begin{gathered}
\nu_{n}=(2 n+1) \frac{\pi}{2}, \quad \mu_{j}=(2 j+1) \frac{\pi}{2}, \\
\Psi_{n j}(x, y)=\cos \left(\nu_{n}(1-x)\right) \cos \left(\mu_{j} y\right),
\end{gathered}
$$

$$
f_{j}(s)=\int_{0}^{1} f(y, s) \cos \left(\mu_{j} y\right) d y, \quad j=0,1, \ldots
$$

Proof. See Appendix B for the proof.

From the lemma we see that for $x=1$ in (3.1) we have

$$
\begin{aligned}
g(y, t) & =u(1, y, t) \\
& =\sum_{j=0}^{\infty}\left(\int_{0}^{t} a \sum_{n=0}^{\infty}(-1)^{n} \nu_{n} \exp \left(-\left(a \nu_{n}^{2}+b \mu_{j}^{2}\right)(t-s)\right) f_{j}(s) d s\right) \cos \left(\mu_{j} y\right) .
\end{aligned}
$$

Copyright ( $\odot$ by SIAM. Unauthorized reproduction of this article is prohibited. 
We now expand $g(y, t)$ in the same cosine series,

$$
g(y, t)=\sum_{j=0}^{\infty} g_{j}(t) \cos \left(\mu_{j} y\right) .
$$

Since the functions $\cos \left(\mu_{j} y\right)$, for $j=0,1, \ldots$, are orthogonal with respect to the inner product $(f, g)=\int_{0}^{1} f(y) g(y) d y$, comparing (3.5) and (3.4) leads to

$$
g_{j}(t)=\int_{0}^{t} a \sum_{n=0}^{\infty}(-1)^{n} \nu_{n} \exp \left(-\left(a \nu_{n}^{2}+b \mu_{j}^{2}\right)(t-s)\right) f_{j}(s) d s .
$$

Therefore, the solution at the left boundary $f(y, t)$ can be computed formally from the given data function $g(y, t)$ at the right, by the following procedure.

1. Compute the cosine transform of the data function:

$$
g_{j}(t)=\int_{0}^{1} g(y, t) \cos \left(\mu_{j} y\right) d y, \quad j=0,1, \ldots
$$

2. Solve the Volterra integral equations

$$
\int_{0}^{t} k_{j}(t-s) f_{j}(s) d s=g_{j}(t), \quad j=0,1, \ldots,
$$

to find the functions $f_{j}$, where the kernel $k_{j}$ is given by

$$
\begin{aligned}
k_{j}(r) & =a \sum_{n=0}^{\infty}(-1)^{n} \nu_{n} \exp \left(-\left(a \nu_{n}^{2}+b \mu_{j}^{2}\right) r\right) \\
& =\exp \left(-b \mu_{j}^{2} r\right) a \sum_{n=0}^{\infty}(-1)^{n} \nu_{n} \exp \left(-a \nu_{n}^{2} r\right) .
\end{aligned}
$$

3. Evaluate the solution $f(y, t)$ by computing the inverse cosine transform:

$$
f(y, t)=\sum_{j=0}^{\infty} f_{j}(t) \cos \left(\mu_{j} y\right) .
$$

Steps 1 and 3 of the procedure are well-posed operations that can be implemented using the cosine transform and its inverse. Ill-posedness occurs only in step 2. The functions (3.8) are the kernel functions corresponding to the following problems; for more details see Appendix B.

$$
\begin{aligned}
w_{t}(x, t) & =a w_{x x}(x, t)-b \mu_{j}^{2} w(x, t), & & 0<x<1, \quad 0 \leq t \leq 1, \\
w(x, 0) & =0, & & 0 \leq x \leq 1, \\
w(1, t) & =g_{j}(t), & & 0 \leq t \leq 1 \\
w_{x}(1, t) & =0, & & 0 \leq t \leq 1 .
\end{aligned}
$$

Consider

$$
g_{j}(t)=\left(K_{j} f_{j}\right)(t)=\int_{0}^{t} k_{j}(t-s) f_{j}(s) d s, \quad 0 \leq t \leq 1 .
$$

Further insight into the properties of the 2D SPE can be obtained by studying numerically the kernel functions $k_{j}$ and the operators $K_{j}$. In Figure 2 we illustrate the kernel functions $k_{j}$ for $j=0,1, \ldots, 8$. First we note that the magnitude of the kernel functions decreases with increasing $j$, and in fact the decrease is exponential; see Figure 3. There we have plotted the first singular value, denoted $\sigma_{1}^{(j)}$, of each operator 

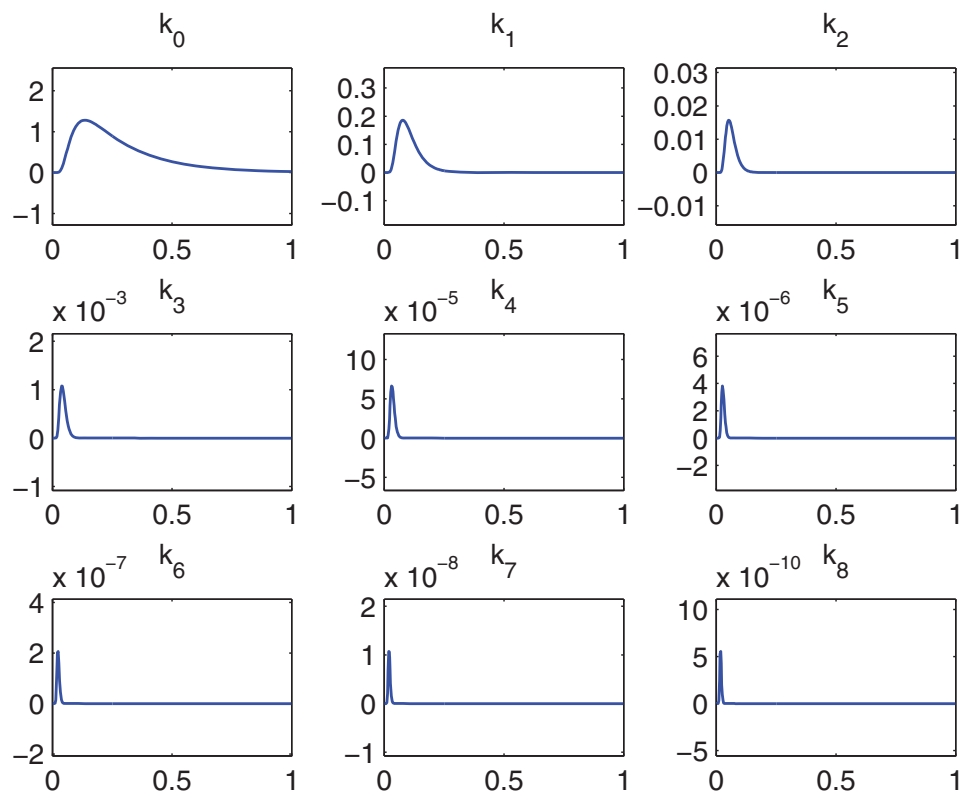

FIG. 2. Kernel functions $k_{j}(r), j=0,1,2, \ldots, 8$.

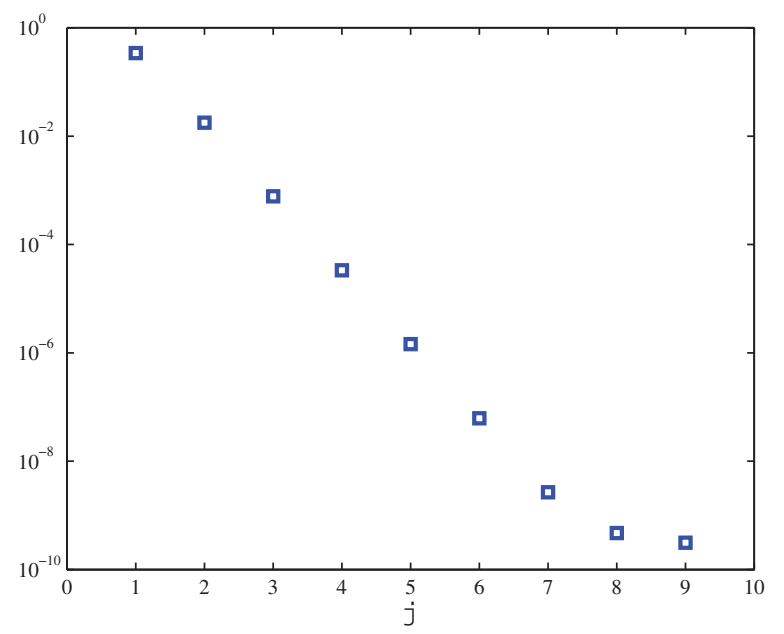

FIG. 3. First singular value $\sigma_{1}^{(j)}$ of each (discretized) operator $K_{j}, j=0,1,2, \ldots, 8$.

$K_{j}$ (discretized to a matrix of dimension 400; since the discretization error is $O\left(h^{2}\right)$, where $h$ is the step length, this is fine enough to ignore the discretization error).

As the kernel functions are smooth, the operators are compact [14, p. 37], with singular values tending to zero. However, in Figure 2 we see that the kernels become less smooth with increasing $j$, which indicates that the Volterra equations (3.7) become less severely ill-posed. The graphs indicate that for large $j$, the kernels behave like Dirac delta functions. This is further illustrated in Figure 4, where we have plotted the first 30 singular values of the normalized operators (matrices) $\widehat{K}_{j}=1 / \sigma_{1}^{(j)} K_{j}$. We see that it is only for the first few operators that there is a substantial decay of the singular values, but for the rest, the decay is very slow. Thus the behavior of the 

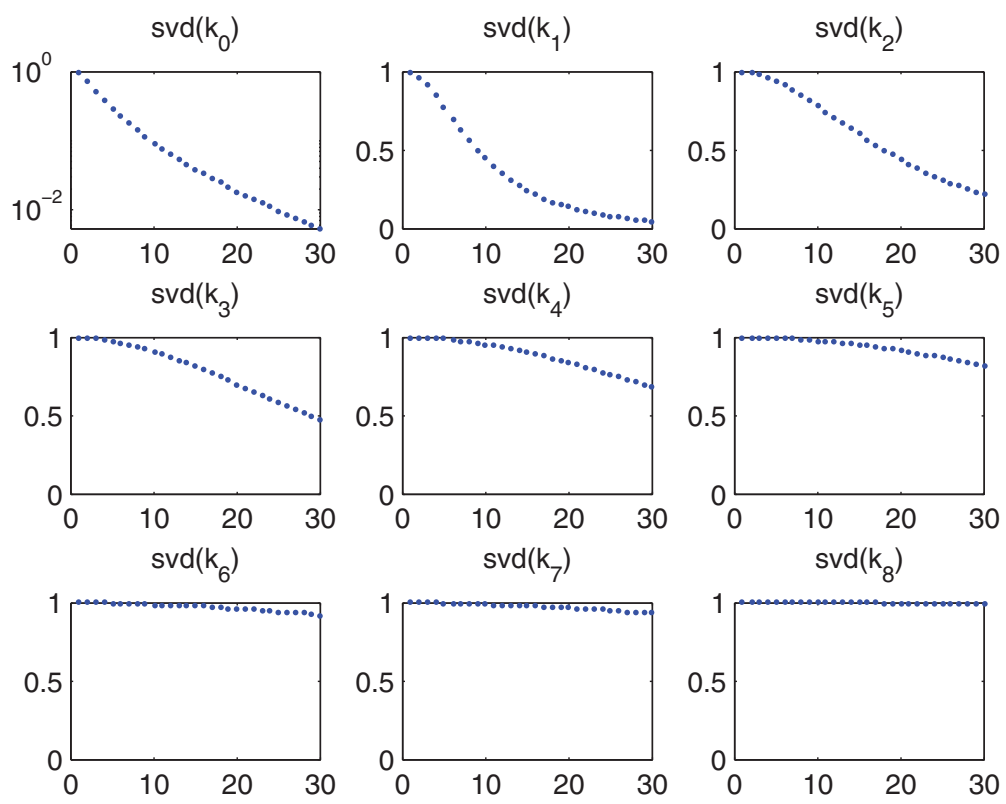

FIG. 4. First 30 singular values of the normalized operators $\widehat{K}_{j}, j=0,1,2, \ldots, 8$.

kernel function in Figure 2 is consistent with the decay of singular values in Figure 4. As a consequence, it is only for the first few Volterra equations that there is really a need to regularize the numerical solution.

We can summarize the above discussion as follows. In terms of normalized operators $\widehat{K}_{j}$, we can write the equations $(3.7)$ as

$$
g_{j}=K_{j} f_{j}=\sigma_{1}^{(j)} \widehat{K}_{j} f_{j}, \quad\left\|\widehat{K}_{j}\right\|=1
$$

where the norm is the operator norm. Let $\widehat{K}_{j}^{\dagger}$ denote a pseudoinverse of the operator $\widehat{K_{j}}$. If we allow ourselves to write the solution of (3.11) formally as

$$
f_{j}=\frac{\widehat{K}_{j}^{\dagger} g_{j}}{\sigma_{1}^{(j)}}
$$

we can now write the solution (3.10), again formally, as

$$
f(y, t)=\sum_{j=0}^{\infty} \frac{\left(\widehat{K}_{j}^{\dagger} g_{j}\right)(t)}{\sigma_{1}^{(j)}} \cos \left(\mu_{j} y\right) .
$$

Obviously, the cosine expansion is in terms of the space variable $y$, while the Volterra equations are models of time-dependent processes. The ill-posedness of the 2D Cauchy problem can be seen to be due to the exponential decay of the $\sigma_{1}^{(j)} \mathrm{s}$ (y-dependence) combined with the ill-posedness of the Volterra equations ( $t$-dependence). The expansion (3.12) together with the analysis of the Volterra operators show that it is only for small $j$ that the $t$-ill-posedness is severe; for larger value of $j$, the $t$-ill-posedness is unimportant.

Of course, the rate of decay of the singular values depends also on the actual values of the coefficients $a$ and $b$. It can be seen from (3.8) that for larger values of 
$a$ and $b$ the kernel functions become less smooth. Similarly, we see from (3.9) that the larger the value of $b$, the more narrow the peak of the kernel function, and the faster the decay of the first singular values $\sigma_{1}^{(j)}$. With fast decay as in Figure 3, only a few of the terms in (3.12) can be included without the approximate solution being dominated by noise. This means that one can only compute approximate solutions that are quite smooth.

3.2. Preconditioner. For the construction of a preconditioner based on the constant coefficient case, we see from (3.12) that to stabilize the solution, we need to regularize the Volterra equations and truncate the cosine expansion.

Let $M$ represent the operator corresponding to (1.1) with constant coefficients, where the constants are chosen as the mean values of the respective variable coefficients in the original problem (1.1). If the variations of the coefficients $a(x, y)$ and $b(x, y)$ are moderate, then $M$ is close to the operator $K$, and it is appropriate to use it as a preconditioner. But obviously $M$ is an operator with unbounded inverse (see also (3.12)), so the preconditioner solve must be regularized. Thus we let the preconditioner be a discrete and truncated version of the solution procedure given in section 3.1. The Volterra equations (3.7) are solved using Tikhonov regularization and a finite number of terms in the series (3.10) are included.

In the preconditioner we have two parameters, which have a regularizing effect. The first is the Tikhonov regularization parameter $\lambda$ in the solution of the Volterra equations (3.7). If the value of $\lambda$ is too small more noise is introduced in the regularized solution and if the value of $\lambda$ is too large then we may suppress smooth solution components that are relevant. For the choice of $\lambda$, see the numerical experiments below.

The number of terms, $p$, in the approximation of the series (3.10), also acts as a regularization parameter. We saw in section 3 that the magnitude of the kernels of the operators $K_{j}$ decreases exponentially with increasing $j$. Therefore, it is necessary to truncate the series, because otherwise they would lead to blow up of noise; cf. (3.12). Due to the fast decay, only a small number of terms need be included (this also affects how nonsmooth solutions can be computed). In our numerical experiment in section 4 it turned out that the choice of $p$ was not critical: if we took a few more terms than warranted by the decay of the kernels it did not change the quality of the solution. Note that Tikhonov regularization and truncation of the expansion are used only as auxiliary regularization methods to construct the preconditioner, whose action is corrected by the GMRES iterations.

The implementation of the preconditioner $M_{r}^{\dagger} v=u$ is summarized in the following algorithm. To represent the functions $v(y, t)$ and $u(y, t)$ discretized on a regular grid $\left(y_{i}, t_{j}\right), i=1, \ldots, m, j=1, \ldots, n$, we use the matrices $V \in \mathbb{R}^{m \times n}$ and $U \in \mathbb{R}^{m \times n}$, respectively. The rank of the preconditioner $r=p m$.

Algorithm. Preconditioner Solve. Given $V$, $p$, and $\lambda$, the following algorithm computes $u=M_{r}^{\dagger} v$.

1. Discrete cosine transform of $V$ applied to each column (i.e., in $t$ direction) (see $(3.6)$ ), $\hat{V}=\operatorname{odct}(V) .^{3}$

2. Solve the Volterra integral equations (3.7),

$$
\text { for } \begin{aligned}
j=1: p \\
\hat{V}_{j}=\hat{V}(j,:)^{T} .
\end{aligned}
$$

\footnotetext{
${ }^{3}$ Compute the (inverse) discrete cosine transform of a vector or the columns of a matrix differ from dct MATLAB function in the way that we transform only with odd frequencies; see (3.2).
} 

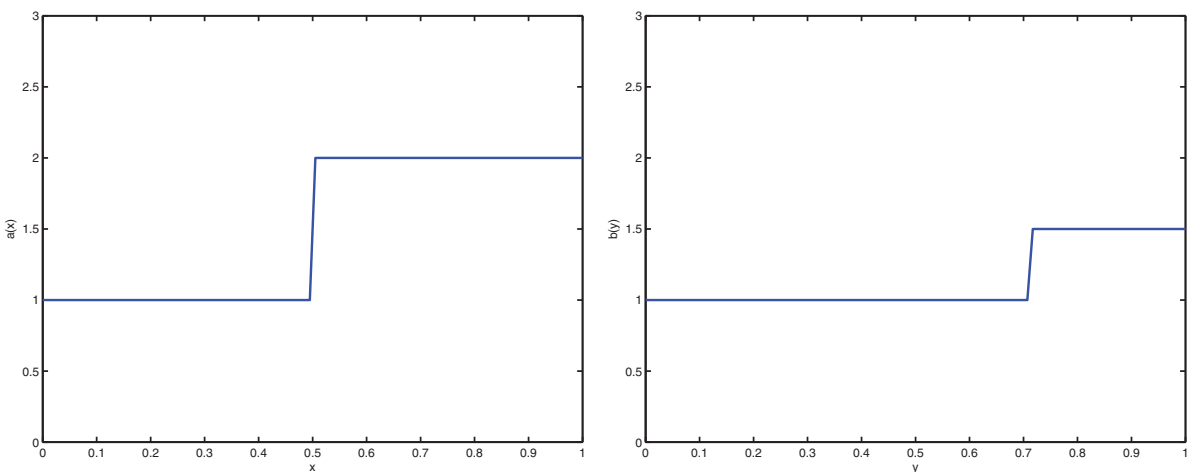

Fig. 5. Plot of variable coefficients a(x) (left) and b(y) (right).

Solve $K_{j} \hat{U}_{j}=\hat{V}_{j}$, using Tikhonov regularization (3.13). Here $K_{j}$ is the discretized integral operator with the kernel $k_{j}$ in (3.8).

$\hat{U}=\left(\begin{array}{c}\hat{U}_{1} \\ \hat{U}_{2} \\ \vdots \\ \hat{U}_{p} \\ 0 \\ \vdots \\ 0\end{array}\right)$.

4. Evaluate the solution by computing the inverse discrete cosine transform applied to each column in (3.10).

$U=\operatorname{iodct}(\hat{U})$.

Let $L$ be the discretization of a differentiation operator. The solution of the least square problems $\min _{\hat{U}_{j}}\left\|K_{j} \hat{U}_{j}-\hat{V}_{j}\right\|_{2}$ by Tikhonov regularization, i.e., the solution of

$$
\min _{\hat{U}_{j}}\left\|\left(\begin{array}{c}
K_{j} \\
\lambda L
\end{array}\right) \hat{U}_{j}-\left(\begin{array}{c}
\hat{V}_{j} \\
0
\end{array}\right)\right\|_{2}
$$

for every $j=1, \ldots, p$, can be computed efficiently: As the kernel function in the Volterra integral equation (3.7) is shift invariant, the discretization will be a Toeplitz matrix. Also $L$ will be Toeplitz. The solution of (3.13) can be computed using plane rotations in $O\left(n^{2}\right)$ floating point operations, where $n \times n$ is the dimension of $K_{j}$, using the algorithm in [11].

Each application of the preconditioner requires $O(m \log m)+O\left(\mathrm{pn}^{2}\right)$ floating point operations. In our MATLAB experiments the time for the preconditioner was less than $0.1 \%$ of the total time consumed for solving the entire problem. Most of the time is used for solving the well-posed 2D problem in each GMRES iteration.

4. Numerical experiments. In this section we present two numerical examples intended to demonstrate that the proposed method works well. For both examples we select the variable coefficients $a$ and $b$ as illustrated in Figure 5. For simplicity, we let $a(x)$ and $b(y)$ be functions of one spatial variable only.

The examples were constructed as follows: First we selected a solution $u(0, y, t)=$ $f(y, t)$. In order to find $u(1, y, t)=g(y, t)$, the associated well-posed problem (2.4) 
was solved using a method of lines. First we discretize the space derivatives, on two equidistant grids, $0 \leq x_{1}<\cdots<x_{q} \leq 1,0 \leq y_{1}<\cdots<y_{m} \leq 1$, and let $U_{i, j}(t)$ denote the approximate value of $u\left(x_{i}, y_{j}, t\right)$. Accordingly we obtain a system of ODEs $U_{t}=A U$, where the matrix $\mathrm{A}$ is large of dimension $(m q \times m q)$ and sparse. For both examples we choose $m=q=40$. The ODE is then solved using MATLAB routine ode23s.

Normally distributed noise was added giving a noisy function $g_{\delta}=g+\delta$ such that for all examples the relative perturbation was 5\% (measured in the Euclidean vector norm).

Given the perturbed data function, the PGMRES method was applied to reconstruct the function $f$. To stop the iterations we used the discrepancy principle, i.e., we stopped the iteration when the residual,

$$
\left\|K f^{(k)}-g_{\delta}\right\| \leq \delta .
$$

In the experiments below we used the value of $\delta$ from the construction of the perturbed data. ${ }^{4}$ The preconditioner $M$ was constructed as in section 3.2. In our computational examples we used a fixed $\lambda$ for all regularization problems (3.13) at all steps of GMRES. To find the best regularization parameter $\lambda$, we selected the smallest $\lambda$ which gave a smooth solution for solving $M z=g_{\delta}$ using the algorithm in section 3.2. The parameter $p$, i.e., the number of terms in the cosine expansion of the solution, was found by trial and error. In our experiments very few terms were needed. In the examples below a slightly larger value $p$ did not change the solution visibly.

Example 1. In our first example the solution function is,

$$
f(y, t)=\operatorname{erf}(5(1-y)) T(t)
$$

where

$$
T(t)= \begin{cases}0, & 0 \leq t \leq 1 / 4 \\ 75(t-0.25)^{2}, & 1 / 4<t \leq 7 / 20 \\ 0.75+(20 t-7)(8-20 t), & 7 / 20<t \leq 2 / 5 \\ 0.75 \exp (2(8-20 t)), & 2 / 5<t \leq 3 / 4 \\ 0, & 3 / 4<t \leq 1\end{cases}
$$

We used $\lambda=0.03$ and $p=2$ to get a good solution. In the Tikhonov regularization (3.13), the matrix $L$ was the discretization of the first derivative operator. The results are displayed in Figures 6-8. The results given in Figure 7 are quite satisfactory after 10 iterations of PGMRES and are comparable to those obtained with standard methods for ill-posed parabolic problems; see, e.g., [12].

Example 2. In order to demonstrate the method is not restricted to the particular boundary conditions in (1.1), we next assumed

$$
u(x, 0, t)=0=u(x, 1, t) .
$$

In order to accommodate this assumption we used an expansion in terms of sines in (3.6) instead of cosines. Thus a discrete sine transform dst replaced odct in the

\footnotetext{
${ }^{4}$ In actual thermal engineering problem one often has a rather good knowledge of the accuracy of the measurements.
}

Copyright $@$ by SIAM. Unauthorized reproduction of this article is prohibited. 

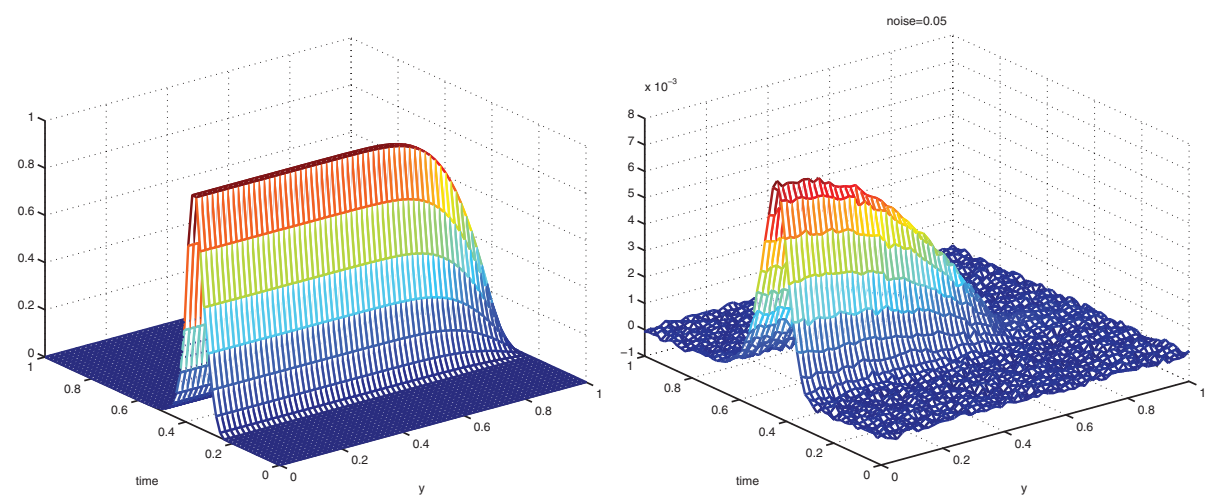

FiG. 6. Example 1. Exact solution $f(y, t)$ (left) and data function $g_{\delta}(y, t)$ with $5 \%$ noise (right).
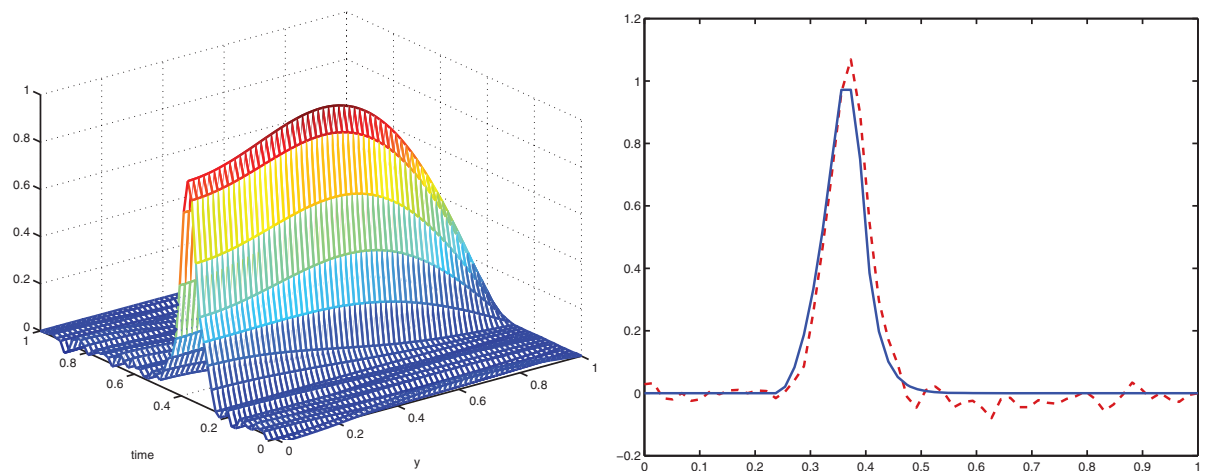

FIG. 7. Example 1. Approximate solution (left), and the exact solution (solid) and the approximate solution (dashed) at $y=0$ (right) after 10 iterations of PGMRES with $5 \%$ perturbation in the data. We used $\lambda=0.03$ and $p=2$.
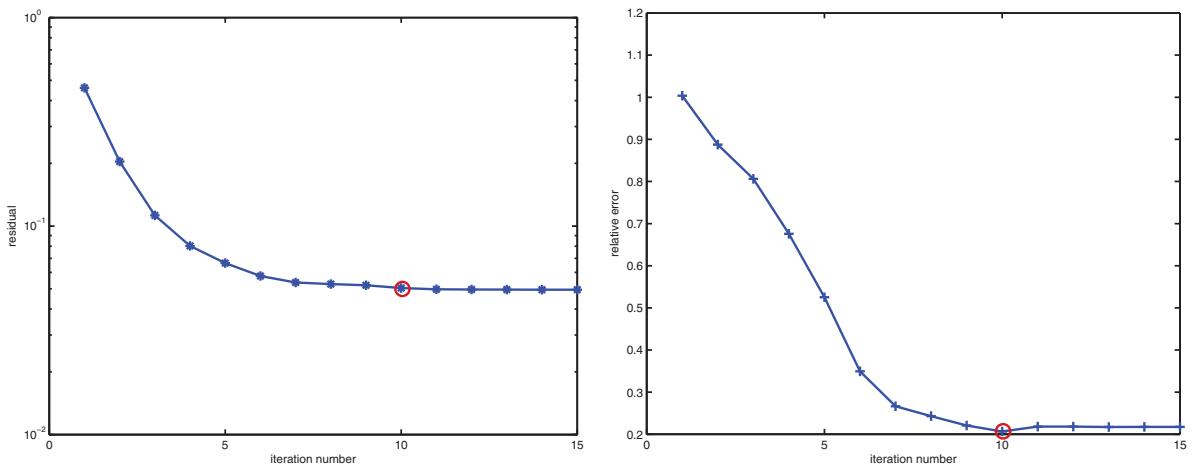

FIG. 8. Example 1. Relative residuals (left) and errors (right) of PGMRES as functions of the iteration index. The circles mark when the stopping criterion was first satisfied.

algorithm. The solution was chosen as

$$
f(y, t)=\exp \left(4-\frac{1}{y(1-y)}\right) \exp \left(4-\frac{1}{t(1-t)}\right) .
$$

Copyright (c) by SIAM. Unauthorized reproduction of this article is prohibited. 

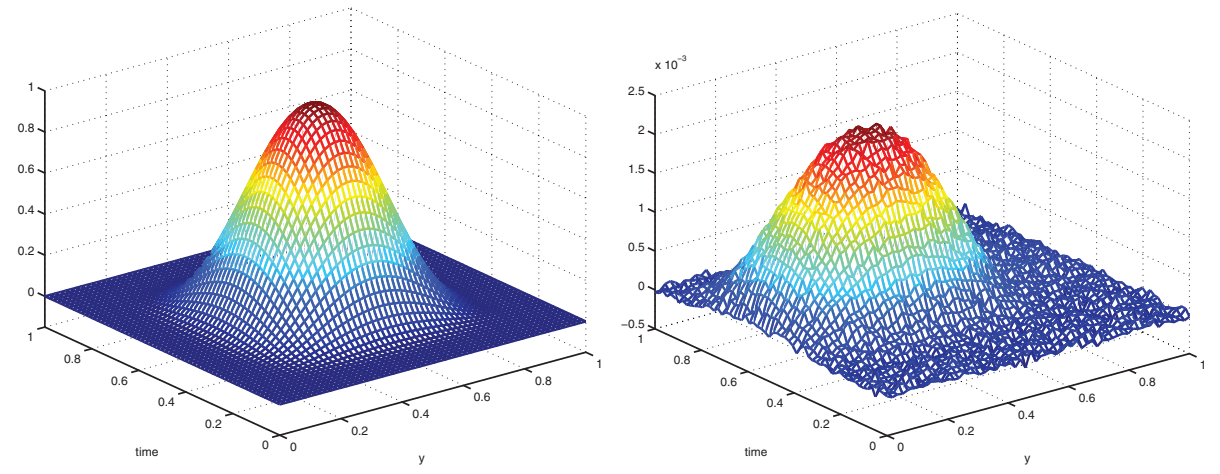

FIG. 9. Example 2. Exact solution $f(y, t)$ (left) and data function $g_{\delta}(y, t)$ with $5 \%$ noise (right).
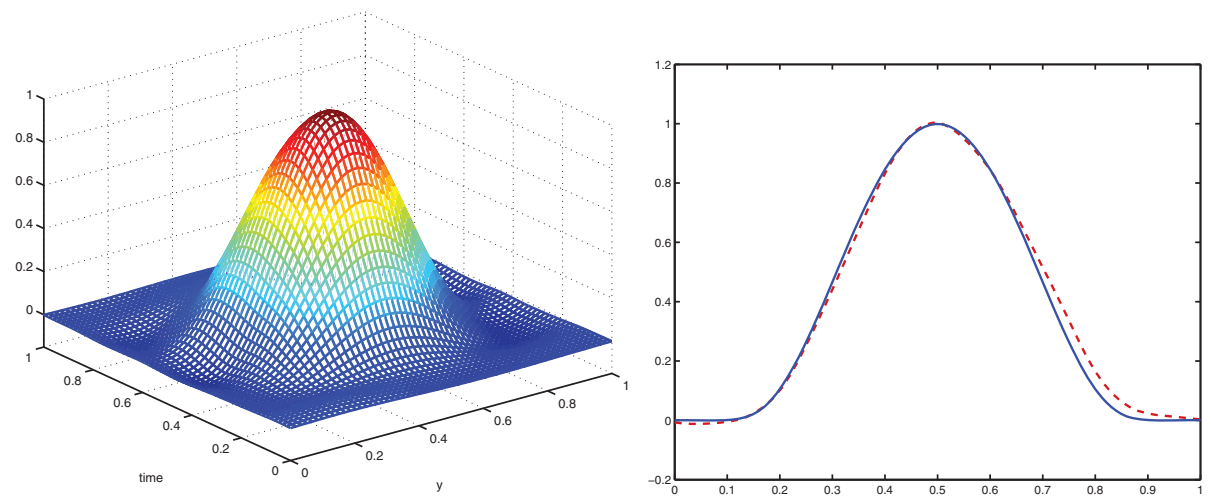

FIG. 10. Example 2. Approximate solution (left) and the exact solution (solid) and the approximate (dashed) at $y=1 / 2$ (right) after 16 iterations of PGMRES with $5 \%$ perturbation in the data. We used $\lambda=0.04$ and $p=3$.
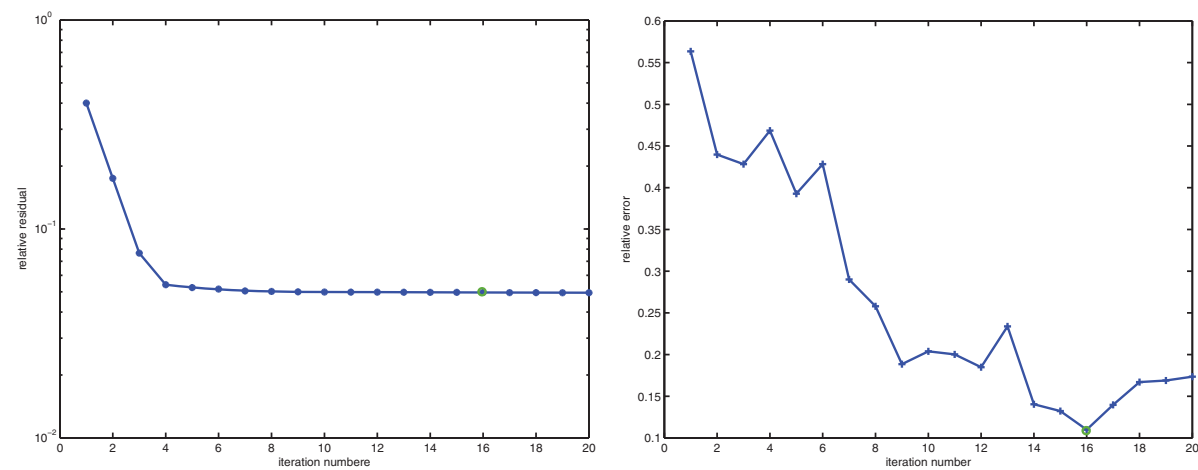

FIG. 11. Example 2. Relative residuals (left) and relative errors (right) of PGMRES as function of the iteration index. The circles mark when the stopping criterion was first satisfied.

$\lambda=0.04$ and $p=3$ gave the best result. The matrix $L$ in (3.13) was the discretization of the second derivative operator. The approximate solution was obtained after 16 iterations of PGMRES. The results are displayed in Figures 9-11. This problem is difficult: Due to the closeness of the solution function to zero in the vicinity of 0 and 
1, which can be seen in Figure 10, it is not possible to approximate the solution in terms of a small number of sine functions.

5. Conclusions. In this paper we have presented a new iterative regularization technique for solving a 2D SPE with variable coefficients using PGMRES.

The preconditioner is the approximation of the 2D SPE problem with constant coefficients. An important and novel feature of the preconditioner is that it is singular, and based on a truncated expansion in terms of trigonometric functions. Therefore a pseudoinverse is used. Applying the regularized preconditioner gives a good approximation of the low frequency part of the solution. As the preconditioner is formulated in terms of trigonometric functions, it can be implemented using fast discrete trigonometric transforms, in combination with the solution of a relatively small number of simple 1D sideways heat problems. A useful advantage of the right PGMRES method is that we can obtain the residual norm very cheaply, thereby avoiding having to solve an extra 2D well-posed parabolic problem in each iteration to find the residual.

In other areas, where iterative methods are used to solve ill-posed problems, preconditioners are usually constructed by approximating the operator $K$ in (2.1) by another operator $M$, for which a fast solver is available; see, e.g., [17]. In our setting, where $K$ is not known explicitly, we can still use an analogous approach by approximating the underlying differential operator in such a way that a nearby differential equation can be solved quickly (provided it is regularized).

The proposed method is an example of a hybrid regularization method [31, 2], $[19$, p. 129]. Regularizing the preconditioner stabilizes the convergence behavior of the PGMRES iteration and makes the quality of the solution less sensitive to the choice of regularization parameter (the number of PGMRES steps). Thus, as we can consider the preconditioner as an approximate solution operator, whose action is corrected using the GMRES iteration (section 2.2), the iterative method is the main regularization method, and the overall method becomes quite insensitive to the choice of preconditioner parameters.

The main focus of the paper has been to investigate a method for the solution of problems, 2D (in space) and with variable coefficients, that arise, e.g., in thermal engineering. Thus, we have not shown that the proposed method is a regularizing method in the strict sense described, e.g., in [14, Chapter 3]. This is because the algorithm in [13], on which the method of the present paper is based, is derived in a finite-dimensional setting, using the Schur decomposition that, to our knowledge, has no counterpart in the infinite-dimensional case. Our results indicate that the method can be used for even more general problems (e.g., in three space dimensions with complicated geometry), provided that a stabilized, efficient preconditioner can be devised and there is an efficient solver for the fully general corresponding well-posed problem. We conjecture that it is possible for a less general problem formulation to extend the theory of [13] and prove strictly that the proposed method is a regularizing method, at least in the self-adjoint, stationary case. However, that may entail rather strict assumptions on the coefficients of the PDE that will limit the usefulness for realistic problems from applications.

Future work includes extension to general multidimensional problems, and the investigation of the use of singular preconditioners for the solution of other ill-posed PDE problems with Krylov methods.

Appendix A. Ill-posedness. We demonstrate here the ill-posedness of the Cauchy problem (1.1) for constant coefficients $a=b=1$. For another example with slightly different data, see [29]. Obviously the qualitative properties are the same for 
nonconstant coefficients. First we define all functions to be zero for $y<0, y>1$ and also for $t<0, t>1$. Suppose that $\xi$ and $\tau$ represent the Fourier transform variables associated with $y$ and $t$. Let

$$
\widehat{g}(\xi, \tau)=\frac{1}{2 \pi} \int_{-\infty}^{\infty} \int_{-\infty}^{\infty} g(y, t) e^{-i \xi y} e^{-i \tau t} d y d t
$$

be the Fourier transform of the exact data function. The problem (1.1) can now be formulated, as follows:

$$
\begin{aligned}
\widehat{u_{x x}}(x, \xi, \tau) & =\left(i \tau+\xi^{2}\right) \widehat{u}(x, \xi, \tau), & & 0<x<1, \quad(\xi, \tau) \in \mathbb{R}, \\
\widehat{u}(1, \xi, \tau) & =\widehat{g}(\xi, \tau), & & (\xi, \tau) \in \mathbb{R}, \\
\widehat{u_{x}}(1, \xi, \tau) & =0, & & (\xi, \tau) \in \mathbb{R} .
\end{aligned}
$$

The solution to this problem is given by

$$
\widehat{u}(x, \xi, \tau)=\widehat{g}(\xi, \tau) \cosh (\alpha(1-x)),
$$

where

$$
\alpha=\sqrt{i \tau+\xi^{2}}=\frac{1}{\sqrt{2}}\left[\left(\sqrt{\tau^{2}+\xi^{4}}+\xi^{2}\right)^{1 / 2}+i \operatorname{sign}(\tau)\left(\sqrt{\tau^{2}+\xi^{4}}-\xi^{2}\right)^{1 / 2}\right] .
$$

Since the real part of $\alpha$ is positive the error in the high frequency components of $\widehat{g}$ is amplified by the factor $\exp \left(\left(\left(\sqrt{\tau^{2}+\xi^{4}}+\xi^{2}\right) / 2\right)^{1 / 2}\right)$, where both $\xi$ and $\tau$ tend to infinity. Thus the inverse problem is severely ill-posed, in fact more ill-posed than the Cauchy problem in one space variable. For detailed Fourier analysis of equation $(1.1)$, see $[32,29]$.

\section{Appendix B. Proof of Lemma 3.1.}

Proof. Taking into account the homogeneous boundary conditions at $y=0$ and $y=1$ we try to find the solution (1.1) with constant coefficients in the form

$$
u(x, y, t)=\sum_{j=0}^{\infty} u_{j}(x, t) \cos \left(\mu_{j} y\right), \quad \mu_{j}=(2 j+1) \pi / 2,
$$

where $u_{j}(x, t)$ are to be determined. For this purpose we expand $f(y, t)$ in the same kind of Fourier series,

$$
f(y, t)=\sum_{j=0}^{\infty} f_{j}(t) \cos \left(\mu_{j} y\right)
$$

Let

$$
w:=u_{j}
$$

Plugging (B.1) into (1.1), we get

$$
\sum_{j=0}^{\infty} \cos \left(\mu_{j} y\right)\left(a w_{x x}(x, t)-b \mu_{j}^{2} w(x, t)-w_{t}(x, t)\right)=0 .
$$

Thus

$$
w_{t}(x, t)=a w_{x x}(x, t)-b \mu_{j}^{2} w(x, t)
$$

Copyright ( $\odot$ by SIAM. Unauthorized reproduction of this article is prohibited. 
On the other hand

$$
w(x, 0)=0, \quad w(0, t)=f_{j}(t), \quad w_{x}(1, t)=0 .
$$

Consequently, we can find $w(x, t)$ by solving the following PDE,

$$
\begin{aligned}
w_{t}(x, t) & =a w_{x x}(x, t)-b \mu_{j}^{2} w(x, t), & & 0<x<1, \quad 0 \leq t \leq 1, \\
w(x, 0) & =0, & & 0 \leq x \leq 1 \\
w(0, t) & =f_{j}(t), & & 0 \leq t \leq 1 \\
w_{x}(1, t) & =0, & & 0 \leq t \leq 1
\end{aligned}
$$

Now let

$$
v(x, t)=\exp \left(b \mu_{j}^{2} t\right) w(x, t),
$$

then we have the following 1D (in space) SPE,

$$
\begin{aligned}
v_{t}(x, t) & =a v_{x x}(x, t), & & 0<x<1, \quad 0 \leq t \leq 1 \\
v(x, 0) & =0, & & 0 \leq x \leq 1 \\
v(0, t) & =\exp \left(b \mu_{j}^{2} t\right) f_{j}(t), & & 0 \leq t \leq 1 \\
v_{x}(1, t) & =0, & & 0 \leq t \leq 1
\end{aligned}
$$

By Appendix C we have

$$
v(x, t)=a \int_{0}^{t} \sum_{n=0}^{\infty}(-1)^{n} \nu_{n} \exp \left(-a \nu_{n}^{2}(t-s)\right) \cos \left((1-x) \nu_{n}\right) \exp \left(b \mu_{j}^{2} s\right) f_{j}(s) d s .
$$

Further by (B.6) and (B.5)

$$
w(x, t)=a \int_{0}^{t} \sum_{n=0}^{\infty}(-1)^{n} \nu_{n} \exp \left(-\left(a \nu_{n}^{2}+b \mu_{j}^{2}\right)(t-s)\right) \cos \left((1-x) \nu_{n}\right) f_{j}(s) d s .
$$

A combination of (B.7), (B.3), (B.1) gives the result of the lemma.

Appendix C. 1D SPE. Consider the following 1D SPE,

$$
\begin{aligned}
u_{t}(x, t) & =a u_{x x}(x, t), & & 0<x<1, \quad 0 \leq t \leq 1, \\
u(x, 0) & =0, & & 0 \leq x \leq 1, \\
u(0, t) & =f(t), & & 0 \leq t \leq 1, \\
u_{x}(1, t) & =0, & & 0 \leq t \leq 1 .
\end{aligned}
$$

Let

$$
w(x, t)=u(x, t)-f(t)
$$

and $f(0)=0$, then we have

$$
\begin{aligned}
w_{t}(x, t) & =a w_{x x}(x, t)-f^{\prime}(t), & & 0<x<1, \quad 0 \leq t \leq 1 \\
w(x, 0) & =0, & & 0 \leq x \leq 1 \\
w(0, t) & =0, & & 0 \leq t \leq 1 \\
w_{x}(1, t) & =0, & & 0 \leq t \leq 1 .
\end{aligned}
$$

Separation of variables and homogeneous boundary conditions lead to

$$
w(x, t)=\sum_{k=0}^{\infty} \alpha_{k}(t) \sin \left(\nu_{k} x\right)=\sum_{k=0}^{\infty} \alpha_{k}(t)(-1)^{k} \cos \left(\nu_{k}(1-x)\right)
$$

Copyright (C) by SIAM. Unauthorized reproduction of this article is prohibited. 
with $\nu_{k}=(2 k+1) \pi / 2$. Substituting (C.4) in (C.3) gives

$$
a \nu_{k}^{2} \alpha_{k}(t)+\alpha_{k}^{\prime}(t)=\beta_{k} f^{\prime}(t), \quad \alpha_{k}(0)=0,
$$

where $\beta_{k}=\left\langle 1, \sin \left(\nu_{k} x\right)\right\rangle=1 / \nu_{k}$. The solution of this equation has the form

$$
\alpha_{k}(t)=-\beta_{k} \int_{0}^{t} \exp \left(-a \nu_{k}^{2}(t-s)\right) f^{\prime}(s) d s
$$

By partial integration we have

$$
\begin{aligned}
\int_{0}^{t} \exp \left(-a \nu_{k}^{2}(t-s)\right) f^{\prime}(s) d s= & f(t)-f(0) \exp \left(-a \nu_{k}^{2} t\right) \\
& -a \nu_{k}^{2} \int_{0}^{t} \exp \left(-a \nu_{k}^{2}(t-s)\right) f(s) d s
\end{aligned}
$$

which results in

$$
\begin{aligned}
w(x, t) & =\sum_{k=0}^{\infty}\left(-\beta_{k} f(t)+\beta_{k} a \nu_{k}^{2} \int_{0}^{t} \exp \left(-a \nu_{k}^{2}(t-s)\right) f(s) d s\right) \sin \left(\nu_{k} x\right) \\
& =-f(t)+\sum_{k=0}^{\infty} a \nu_{k} \int_{0}^{t} \exp \left(-a \nu_{k}^{2}(t-s)\right) f(s) \sin \left(\nu_{k} x\right) d s .
\end{aligned}
$$

Further the solution of the problem (C.1) is

$$
\begin{aligned}
u(x, t) & =w(x, t)+f(t) \\
& =\sum_{k=0}^{\infty} a \nu_{k} \int_{0}^{t} \exp \left(-a \nu_{k}^{2}(t-s)\right) f(s) \sin \left(\nu_{k} x\right) d s \\
& =\int_{0}^{t} \sum_{k=0}^{\infty} a \nu_{k} \exp \left(-a \nu_{k}^{2}(t-s)\right) f(s) \cos \left(\nu_{k}(1-x)\right)(-1)^{k} d s .
\end{aligned}
$$

\section{REFERENCES}

[1] J. V. Beck, B. Blackwell, and S. R. Clair, Inverse Heat Conduction. Ill-Posed Problems, Wiley, New York, 1985.

[2] Å. BJÖRCK, A bidiagonalization algorithm for solving ill-posed systems of linear equations, BIT, 28 (1988), pp. 659-670.

[3] E. Blom, P. Nyqvist, And D. Loyd, Suction pyrometer analysis of the instrument and guide for users, Varmeforsk, Stockholm, 2004 (in Swedish).

[4] P. Brianzi, P. Favati, O. Menchi, and F. Romani, A framework for studying the regularizing properties of Krylov subspace methods, Inverse Problems, 22 (2006), pp. 1007-1021.

[5] A. M. Bruaset, A survey of preconditioned iterative methods, Pitman Res. Notes Math. Ser. 328, Longman Scientific \& Technical, Harlow, England, 1995.

[6] D. Calvetti, B. Lewis, and L. Reichel, GMRES-type methods for inconsistent systems, Linear Algebra Appl., 316 (2000), pp. 157-169.

[7] D. Calvetti, B. Lewis, And L. Reichel, GMRES, l-curves, and discrete ill-posed problems, BIT, 42 (2002), pp. 44-65.

[8] D. Calvetti, B. LeWIS, And L. Reichel, On the regularizing properties of the GMRES method, Numer. Math., 91 (2002), pp. 605-625.

[9] A. CARAsso, Determining surface temperatures from interior observations, SIAM J. Appl. Math., 42 (1982), pp. 558-574.

[10] H. Egger, Yi Heng, W. Marquardt, and A. Mhamdi, Efficient solution of a threedimensional inverse heat conduction problem in pool boiling, Inverse Problems, 25 (2009), 095006.

Copyright $@$ by SIAM. Unauthorized reproduction of this article is prohibited. 
[11] L. EldÉn, An efficient algorithm for the regularization of ill-conditioned least squares problems with triangular Toeplitz matrix, SIAM J. Sci. Stat. Comput., 5 (1984), pp. 229-236.

[12] L. Eldén, F. Berntsson, and T. Reginska, Wavelet and Fourier methods for solving the sideways heat equation, SIAM J. Sci. Comput., 21 (2000), pp. 2187-2205.

[13] L. Eldén And V. Simoncini, Solving ill-posed linear systems with GMRES and a singular preconditioner, SIAM J. Matrix Anal. Appl., 33 (2012), pp. 1369-1394.

[14] H. Engl, M. Hanke, and A. Neubauer, Regularization of Inverse Problems, Kluwer Academic, Dordrecht, The Netherlands, 1996.

[15] L. Guo And D. Murio, A mollified space-marching finite-difference algorithm for the twodimensional inverse heat conduction problem with slab symmetry, Inverse Problems, 7 (1991), pp. 247-259.

[16] E. Hairer And G. WANner, Solving Ordinary Differential Equations II, Springer, Berlin, 1991.

[17] M. Hanke, J. Nagy, and R. Plemmons, Preconditioned iterative regularization for ill-posed problems, in Numerical Linear Algebra, L. Reichel, A. Ruttan, and R. S. Varga eds., de Gruyter, Berlin, 1993, pp. 141-163.

[18] P. C. Hansen, Rank-Deficient and Discrete Ill-Posed Problems. Numerical Aspects of Linear Inversion, SIAM Monogr. Math. Model. Comput., SIAM, Philadelphia, 1997.

[19] P. C. Hansen, Discrete Inverse Problems: Insight and Algorithms, Fund. Algorithms, SIAM, Philadelphia, 2010.

[20] P. C. Hansen and T. Koldborg Jensen, Smoothing-norm preconditioning for regularizing minimum-residual methods, SIAM J. Matrix Anal. Appl., 29 (2006), pp. 1-14.

[21] D. N. HÀo, A. Schneiders, And H. J. Reinhardt, Regularization of a non-characteristic Cauchy problem for a parabolic equation, Inverse Problems, 11 (1995), pp. 1247-1264.

[22] S. Holmgren and K. Отto, Semicirculant solvers and boundary corrections for first-order partial differential equations, SIAM J. Sci. Comput., 17 (1996), pp. 613-630.

[23] T. K. Jensen And P. C. HAnsen, Iterative regularization with minimum-residual methods, BIT, 47 (2007), pp. 103-120.

[24] M. Kilmer And G. W. StewArt, Iterative regularization and MINRES, SIAM J. Matrix Anal. Appl., 21 (2000), pp. 613-628.

[25] P. Knabner and S. Vessella, Stabilization of ill-posed Cauchy problems for parabolic equations, Ann. Mat. Pura Appl. (4), 149 (1987), pp. 393-409.

[26] Y. Knosowski, E. von Lieres, And A. Schneider, Regularization of a non-characteristic Cauchy problem for a parabolic equation in multiple dimensions, Inverse Problems, 15 (1999), pp. 731-743.

[27] Y. Liu And D. A. Murio, Numerical experiments in 2-D IHCP on bounded domains. Part I: The "interior" cube problem, Comput. Math. Appl., 31 (1996), pp. 15-32.

[28] V. A. Morozov, On the solution of functional equations by the method of regularization, Sov. Math. Dokl., 7 (1966), pp. 414-417.

[29] D. A. Murio, The Mollification Method and the Numerical Solution of Ill-Posed Problems, Wiley, New York, 1993.

[30] F. NATTERER, The Mathematics of Computerized Tomography, Wiley, New York, 1986.

[31] D. P. O'Leary And J. A. Simmons, A bidiagonalization-regularization procedure for large scale discretizations of ill-posed problems, SIAM J. Sci. Stat. Comput., 2 (1981), pp. 474-489.

[32] Z. QIAN AND C.-L. FU, Regularization strategies for a two-dimensional inverse heat conduction problem, Inverse Problems, 23 (2007), pp. 1053-1068.

[33] Z. QIAN AND Q. ZhANG, Differential-difference regularization for a $2 d$ inverse heat conduction problem, Inverse Problems, 26 (2010), 095015.

[34] Z. Ranjbar And L. EldÉn, A Preconditioned GMRES Method for Solving a Sideways Heat Equation, Technical report LiTH-MAT-R-2010-06-SE, Department of Mathematics, Linköping University, Linköping, Sweden, 2010.

[35] Z. Ranjbar, Numerical Solution of Ill-posed Cauchy Problems for Parabolic Equations, Linköping Studies in Science and Technology, Dissertations, No. 1300, Linköping University, Linköping, Sweden, 2010.

[36] H. J. Reinhardt, A numerical method for the solution of two-dimensional inverse heat conduction problems, Internat. J. Numer. Methods Engrg., 32 (1991), pp. 363-383.

[37] Y. SAAD, Iterative Methods for Sparse Linear Systems, 2nd ed., SIAM, Philadelphia, 2003.

[38] Y. SaAd And M. H. Schultz, GMRES: A generalized minimal residual algorithm for solving nonsymmetric linear systems, SIAM J. Sci. Stat. Comput., 7 (1986), pp. 856-869.

[39] K. SøRli And I. M. SkaAR, Monitoring the wear-line of a melting furnace, in Inverse Problems in Engineering: Theory and Practice, Third Internatioal Conference on Inverse Problems in Engineering, Port Ludlow, WA, ASME, New York, 1999.

Copyright $@$ ㅇ by SIAM. Unauthorized reproduction of this article is prohibited. 
[40] K. Sørli And I. M. SkAar, Sensitivity analysis for thermal design and monitoring problems of refractories, in Proceedings of CHT-04, ICHMT International Symposium on Advances in Computational Heat Transfer, Kirkenes-Bergen, Norway, ASME, New York, 2004.

[41] P. L. Woodfield, M. Monde, and Y. Mitsutake, Implementation of an analytical twodimensional inverse heat conduction technique to practical problems, Internat. J. Heat Mass Transfer, 49 (2006), pp. 187-197.

Copyright $@$ by SIAM. Unauthorized reproduction of this article is prohibited. 